\title{
Visual environment and delay affect cache retrieval accuracy in a food-storing rodent
}

\author{
CYNTHIA L. BARKLEY and LUCLA F. JACOBS \\ University of California, Berkeley, Califormia
}

\begin{abstract}
Many scatter-hoarding species use spatial memory to relocate their food caches. Two factors can affect spatial memory: the availability of landmarks in the environment, and the latency between learning and recall. Using a $2 \times 2$ factorial design, we determined the effect of these factors on cache retrieval accuracy in Merriam's kangaroo rats (Dipodomys merriami). Kangaroo rats cached seeds in an arena under conditions that varied by retrieval interval ( 1 or 10 days) and by number of landmarks (0 or 16 landmarks). After 1 day, they recovered equal proportions of caches in both landmark conditions. After 10 days, they recovered more caches in the 16-landmark condition than in the bare condition. This is the first study to show that landmarks are necessary for accurate cache recovery after long delays. This result is consistent with maze studies that have shown that landmarks reduce proactive interference and that delay increases proactive interference.
\end{abstract}

Scatter-hoarding animals store food by hiding small amounts of food in many scattered locations or caches (Morris, 1962; Vander Wall, 1990), each of which is created with a single deposition of food. Caches are later retrieved by the hoarder; in the case of the Clark's nutcracker (Nucifraga columbiana) or the gray squirrel (Sciurus carolinensis), the retrieval episode may occur 8-11 months after the caching event (Thompson \& Thompson, 1980; Vander Wall \& Hutchins, 1983). Many sites are used only once, to reduce the risk of cache pilferage by competitors (Andersson \& Krebs, 1978; Daly, Jacobs, \& Wilson, 1992; Hampton \& Sherry, 1994), and when cache sites are virtually unlimited, scatter hoarders rarely reuse sites (Jacobs \& Liman, 1991). Each caching event can therefore be uniquely characterized by its location relative to nearby landmarks.

Laboratory experiments have provided evidence that scatter-hoarding birds, such as the black-capped chickadee (Parus atricapillus) and the Clark's nutcracker and mammals, such as the gray squirrel and the Merriam's kangaroo rat (Dipodomys merriami), can remember the precise locations of their scattered caches (Jacobs, 1995; Shettleworth, 1995). Evidence that memory for cache sites is an evolved adaptation comes both from comparative studies of cache retrieval (Balda \& Kamil, 1989; Clayton, 1995)

We thank Jacqui Gasway and Vince Gifford for technical assistance; Jan Randall for donating kangaroo rats to us from her colony; Martin Daly for traps and other field equipment; Al Muth, Mark Fisher, and the Boyd Deep Canyon Desert Research Station for assistance and accommodations during trapping; and Irv Zucker, Pierre Lavenex, William Timberlake, and the two anonymous reviewers for comments on the manuscript. This research was supported by a National Science Foundation Graduate Fellowship to C.L.B., and funded by grants from the National Science Foundation (NSF IBN-9307317) and the University of California to L.F.J. Correspondence should be addressed to C. L. Barkley, Department of Psychology, University of California, Berkeley, CA 94720 (e-mail: cbarkley@uclink.berkeley.edu). and from artificial tests of spatial memory such as the one-trial associative memory task (Brodbeck, Burack, \& Shettleworth, 1992). In both natural and artificial tests of spatial memory, it is clear that scatter-hoarding animals have a remarkable ability to relocate places in space.

A first step to understanding how this precise spatial memory works is to determine what factors can influence the accuracy of the scatter hoarder's retrieval performance. Two factors that have been shown separately to influence spatial memory in artificial tasks such as the radial arm maze are the latency between learning and testing, with retrieval intervals leading to reduced accuracy (Roberts \& Dale, 1981), and visual landmarks in the learning environment, with presence of landmarks leading to better accuracy (Cohen, Reid, \& Chew, 1994). We are including as landmarks all conspicuous, distinct objects in the environment, both distal and near. These factors may also influence accuracy of cache retrieval. It is already clear that many scatter hoarders characterize each cache by the spatial relations between the cache and landmarks; for example, the retrieval accuracy of Clark's nutcrackers is lower when landmarks present during caching are removed before retrieval (Balda \& Turek, 1984). Shifting landmarks near caches also causes both birds and mammals to shift their search for caches to sites located at the same relative distance to the shifted landmarks (Bennett, 1993; Vander Wall, 1982, 1991). In a recent study, Clark's nutcrackers were found to characterize the site of a hidden food item by the relationships between nearby landmarks in the arena (Kamil \& Jones, 1997). Thus, when landmark cues are available, scatter hoarders use these cues to locate caches. Yet no study has addressed the complementary question: Can scatter hoarders accurately recover caches if conspicuous and salient landmarks are not present during caching?

Although retrieval interval by itself has been shown to affect cache retrieval accuracy in Clark's nutcrackers 
(Balda \& Kamil, 1992), and black-capped chickadees (Parus atricapillus) (Hitchcock \& Sherry, 1990), no study has examined the way in which the length of the retrieval interval and the presence of conspicuous landmarks combine to affect memory for cache locations. In the present study, we examine the possible interaction of these factors in the Merriam's kangaroo rat, a nocturnal, scatterhoarding rodent. Merriam's kangaroo rats are small ( $35 \mathrm{~g})$, primarily (though not exclusively) scatter-hoarding, rodents (Jenkins \& Peters 1992; Reynolds, 1958). In the laboratory, they can rely solely on spatial memory to retrieve their caches (Jacobs, 1992).

Using a $2 \times 2$ factorial design, we tested the effect of distinct landmark arrays and retrieval interval on cache retrieval accuracy in Merriam's kangaroo rats. To manipulate visual environment, we tested kangaroo rats either in a bare environment or in an environment with an array of 16 trial-unique landmarks. We chose to manipulate landmarks by using objects placed on the floor of the arena. Most of the previous experiments showing that scatter hoarders also rely on landmarks to orient themselves to caches have been done with within-apparatus objects (Bennett, 1993; Vander Wall, 1982, 1991). To manipulate delay, we tested the kangaroo rats after retrieval intervals of 1 or 10 days. We predicted that the effects of landmark condition would vary as a function of delay, with recovery accuracy declining more after long delays if caches had been made in a bare environment than after the same delay in an environment with an assortment of unique visual landmarks present.

\section{METHOD}

Kangaroo rats were tested in two cache retrieval interval conditions ( 1 day and 10 days, hereafter 1D and 10D) and two visual environment conditions ( 0 and 16 trial-unique landmarks, or $0 \mathrm{LM}$ and $16 \mathrm{LM}$ ). The short interval was the minimum testable retrieval interval for which we could ensure that in all conditions, animals had restricted access to food for the same period of time and would be hungry at the start of testing. The long retrieval interval selected was similar to the longest retrieval interval of 12 days that has been observed under natural conditions (Daly et al., 1992). In order to provide an array of landmarks that would be salient to the animals, trial-unique arrangements were used.

\section{Animals}

The animals completing the experimental procedure were 6 wildcaught Merriam's kangaroo rats ( 4 females, 2 males). These animals were selected from a group of 20 on the basis of their willingness to cache frequently in the experimental situation. Three kangaroo rats ( 3 females) had been trapped in Portal, Arizona, in 1991 or 1992 and maintained in captivity at San Francisco State University. The other animals ( 2 males, 1 female) were trapped as adults in December 1995 near Palm Desert, California. All animals were housed on sand and were observed to cache food in their home cages.

The kangaroo rats were housed singly in $46 \times 24 \mathrm{~cm}$ plastic cages on sand with cotton bedding and a small container (a capped plastic pipe, a glass jar, or a metal can) as a nest chamber. Prior to the start of the experiment, all animals were placed into a reversed $12: 12-\mathrm{h}$ light:dark cycle, the lights being extinguished at $0800 \mathrm{~h}$. All animals were given ad-lib access to a diet of mixed bird seed and rodent chow. Lettuce was provided as a water source. During the ex- periment, subjects were food restricted to $1.5-2 \mathrm{~g}$ of oats $24 \mathrm{~h}$ prior to a caching or retrieval session. Body weight was monitored throughout the experiment, and they were not permitted to fall below $96 \%$ of their free-feeding baseline.

\section{Apparatus}

The arena for caching was a $118 \times 179 \times 47 \mathrm{~cm}$ open box made of black acrylic plastic, enclosed by white plastic curtains suspended from the ceiling and draped inside the arena walls (see Figure 1). A wood collar supported a raised floor in the arena. This floor consisted of eight galvanized steel plates $(45 \times 45 \mathrm{~cm}$ ), divided into two parallel rows of four each, and the rows were separated by a wood divider $(29 \times 179 \mathrm{~cm})$. Each plate contained a $4 \times 4$ array of $4-\mathrm{cm}$ drilled holes. Each hole was fitted with a cup (4- $\mathrm{cm}$ interior diameter; $3.25 \mathrm{~cm}$ deep) that hung snugly from the cup's rim. These cups, filled with sand, constituted 128 potential cache sites. A plastic dish in the center of the divider acted as a feeder during cache sessions.

Landmarks were used in the arena during the pretest trial, habituation trials, and each $16 \mathrm{LM}$ test trial. We chose objects with natural shapes and textures such as rocks, artificial cloth flowers, sticks, and pine cones as well as short $(10-\mathrm{cm})$ lengths of $7.5-\mathrm{cm}$-diameter black plastic pipe sawed in half. The landmarks were placed on the plates between cups, around the edges of the plates, and on the center divider. The landmarks were randomly chosen for each trial from a pool of approximately 100 objects.

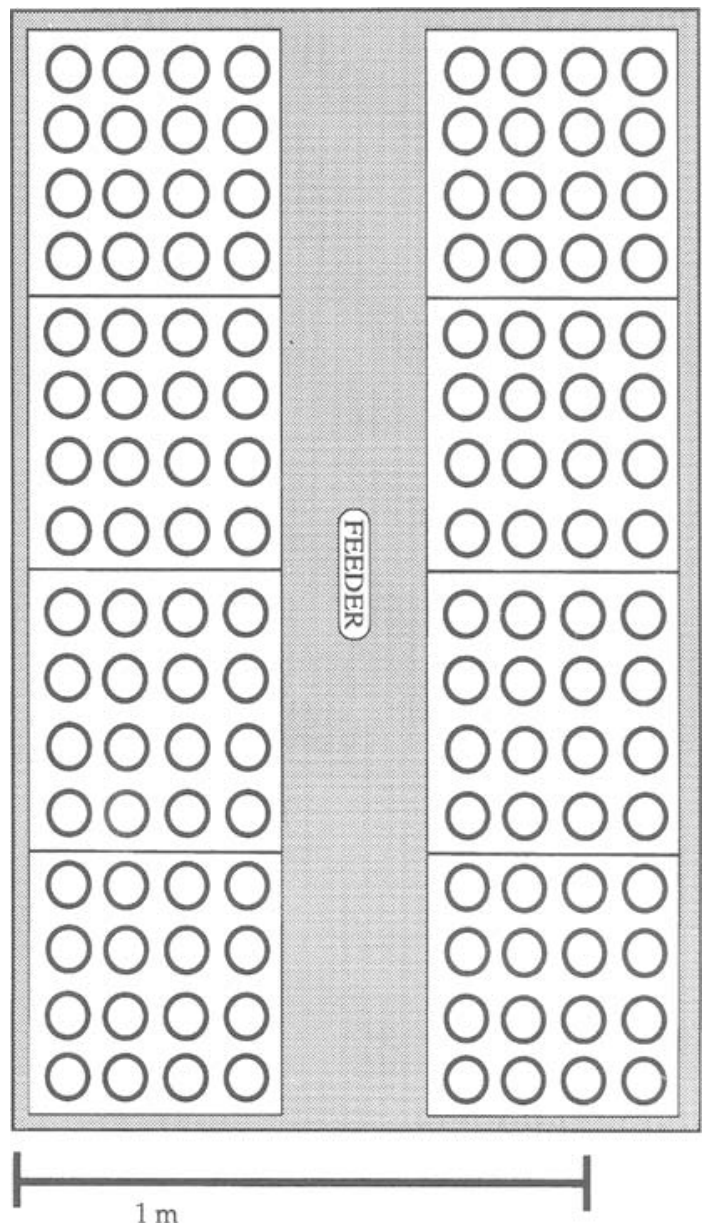

Figure 1. Schematic diagram of the caching arena. The open circles represent the cups suspended from the plates. 
There were some extra-apparatus visual cues that the animals could use to orient themselves in the arena. Holes were cut in the curtains on the two long sides of the arena in order to videotape trials: On one side, a round hole was cut slightly larger than the lens of the camera, and on the other, a large rectangular hole was cut so that a mirror could be suspended at an angle above the arena. This was done because the video camera could not record activity in the entire arena unless it recorded from the mirror. A light also provided an external visual cue. Lighting consisted of a single $60-\mathrm{W}$ bulb on the outside of the curtain beneath the video camera. This light source created a diffuse uneven light in the arena.

\section{Procedure}

The experiment consisted of three phases: pretest, habituation, and test. A trial at each phase of the experiment consisted of two sessions: a cache session and a recovery session. During cache sessions, the feeder in the center of the arena was stocked with 60 sunflower seeds; it was empty during recovery sessions. Eight landmarks were used for trials in the pretest and habituation phases, and either 0 or 16 landmarks were used in the test phase. For each trial, the landmarks were placed in trial-unique configurations, with no landmark occupying a place where one had been in the immediately preceding trial or a place it had ever occupied before. The landmarks were always in the same place in a recovery session as they had been in the preceding cache session.

In the pretest and habituation phases, the animals were checked after $2 \mathrm{~h}$, whereas in the test trials, animals were checked after $20 \mathrm{~min}$. We determined whether the animal had cached by counting the number of seeds left in the feeder, the size of the kangaroo rat's cheek pouches, and whether cups appeared to contain seeds. Two signs indicated that caching had occurred at a site: the presence of sand excavated from a cup and the visible presence of seeds in the cup. Animals were removed from the arena if they had cached seeds in at least two cups.

After the cache sessions, all cups were emptied and the number of seeds cached was recorded. The plates and center divider were wiped with disposable, detergent-impregnated cleaning towels (i.e., "baby wipes") in order to clean or mask odor trails left by the animals. The cups were returned to the plates in new locations. Before retrieval sessions, each cache was replaced. In the pretest phases, all seeds that the animals had cached were returned. In the habituation and test phases, two seeds were returned to each cache, to reduce the likelihood that the animals would become satiated after retrieving one or two caches, and to ensure that all caches emitted the same amount of odor from the seeds. Each cup was covered with a blue plastic poker chip (diameter $=3.75 \mathrm{~cm}$ ), which fit snugly over the sand surface. The chips made searches in cups more obvious and presumably reduced odor cues from the seeds. Once removal was mastered by the animal, the poker chips did not appear to interfere with cache retrieval. All trials were separated by an intertrial interval of at least 11 days, with no more than 15 days elapsing between trials.

\section{Pretest Phase}

Animals were left in the arena with 60 sunflower seeds in the feeder for 2-h blocks until they cached seeds in the sand-filled cups, or until $6 \mathrm{~h}$ had elapsed. Failure to cache on Day 1 resulted in a second caching session the next day for up to $6 \mathrm{~h}$ in duration. The animals that cached were returned to the arena $24 \mathrm{~h}$ later with their caches in cups intact, but with all other seeds (e.g., in the feeder, cached on the plates or on the divider) removed by the experimenter. The kangaroo rats remained in the arena until they had retrieved their caches, or until $6 \mathrm{~h}$ had elapsed. Of the 20 animals pretested, 12 animals cached and recovered sunflower seeds and were retained for the next part of the experiment. Eight landmarks were used during the pretest phase.

\section{Habituation Phase}

Each of the remaining 12 animals was given habituation trials to ensure that it would reliably cache in the cups. The cache sessions of the habituation trials lasted $2 \mathrm{~h}$. A retrieval session occurred on the following day. The retrieval sessions lasted for $80 \mathrm{~min}$ or until the animal had recovered at least $60 \%$ of its caches. Habituation trials continued until the animal had made two caches per session in at least two sessions. The minimum number of habituation trials given to any animal was four. Animals that failed to make two caches in two sessions within seven habituation trials were not used in the remainder of the experiment. Six animals were moved on to the test phase. Of the 6 animals that passed through habituation, 3 had no prior experience. The 3 San Francisco State animals had four to five additional habituation trials in the experimental apparatus 3 months prior to the start of the experiment. Because of the break in training, they were habituated to the arena again.

\section{Test Phase}

The test phase consisted of a $2 \times 2$ factorial within-subjects design. Each animal was tested once in each condition, and the order of conditions was randomly assigned for each subject. The manipulations were delay between cache and recovery (1D or 10D) and the availability of visual cues or landmarks (0LM or $16 \mathrm{LM})$. All test trials were videotaped.

In each cache session, the kangaroo rat was placed in the arena and left for $20 \mathrm{~min}$. The experimenter reentered the room and evaluated whether or not the animal had cached any seeds. The trial ended if the animal had made at least two caches. Otherwise, the trial continued with checks by the experimenter at $35 \mathrm{~min}$ and $50 \mathrm{~min}$. At that point, if the animal had not made two caches, it was returned to its home cage with a ration of oats and the cache session was continued the next day. If the animal made one cache during the 1 st day, that cache was capped with a tightly fitting lid on the 2 nd day. This happened one time for 2 animals.

Recovery sessions occurred either 1 or 10 days after cache sessions. To avoid confusion, the sites used as caches in the previous session will be called target caches. In addition to the target caches, which were each replaced with two seeds, equal numbers of baited control sites, each containing two seeds, were placed in the arena. We chose these sites from those used by the animals themselves as cache sites so that the controls reproduced any site preferences for that individual. The function of the control caches was to ensure that the recovery of target caches was not based solely on search for the odor of seeds. If odor is the exclusive or even the primary cue, kangaroo rats should be equally likely to discover either controls or targets. Control sites were chosen from cups that the animal had used to store seeds in the earliest trials in the arena. On the average, 88 days had elapsed between the animal using the site as a cache and the experimenter choosing it as a control. We estimated the distance between target cache sites and control sites by using the number of cups (either linearly or diagonally) between each control and the closest target and counted the center divider as equivalent to a 2-cup distance. The average distance between each control and the nearest target cache was $8 \pm 0.9$ cups. Recovery sessions lasted $20 \mathrm{~min}$.

All cups in the arena were emptied, and the number of seeds left in each target site or control site were counted. A target or control was considered retrieved if at least one seed had been removed by the animal. The order of sites searched was evaluated from the videotapes of each session. A site was considered searched if an animal had either removed the poker chip and dug in the cup or attempted to remove the poker chip for more than $1.5 \mathrm{sec}$. Searches were categorized as: (1) targets; (2) controls; (3) neighbors, defined as any cup adjacent to a cache site; and (4) uncategorized searches, defined as all other cups in the arena. 


\begin{abstract}
Analysis
Two measures were devised to analyze the data. First, the percentage of target caches and control caches retrieved was calculated. This measure showed the animal's recovery accuracy over the entire session. The second measure, hit percentage, was based on the order of searches. It was designed to be a more precise measure of cache memory by limiting the analysis to the cups first searched by the animal. We analyzed the four categories of search types described above using hit percentage, which we defined as the percentage of searches in each category of the first searches of the session, up to the total number of original caches made in that trial. For example, if a kangaroo rat made four caches, and if it then, during the search of the first four sites, would choose two target sites, one control site and one uncategorized site, the hit percentages would be $50 \%$ target, $25 \%$ control, and $25 \%$ uncategorized sites.
\end{abstract}

\section{RESULTS}

Kangaroo rats made an average of $2.7 \pm 0.2$ caches in each of the four experimental conditions, placing $11.4 \pm$ 0.9 sunflower seeds in each cache. There were no significant differences in the number of seeds placed in each cache per condition. There was a significant effect of visual environment on the number of caches made $[F(1,5)=$ $10.0, p=.025]$; the kangaroo rats made on average one more cache in the $16 \mathrm{LM}$ conditions. Because control sites were chosen from those the animal had previously chosen as cache sites, their history as caches could have potentially affected their probability of retrieval as a control. Eighty-five percent (54/64) of controls were chosen from sites where the cache had been retrieved; of these, $51 \%$ $(28 / 54)$ were retrieved as controls. Of controls chosen from caches that had not been retrieved, $60 \%(6 / 10)$ were retrieved as controls.

Because the percentage of targets or controls recovered could have reflected differences in overall activity level in the arena, the total number of searches that animals made in each condition was calculated. There were 13.3 cups searched per session, on the average. There were no statistically significant differences in total number of cups searched per session [effect of retrieval interval, $F(1,5)=$ $3.73, p=.111$; effect of landmark, $F(1,5)=0.96, p=$ .372 ; interaction, $F(1,5)=1.192, p=.325]$. There was considerable variability between sessions, however (mean number of cups searched per condition: 1D, OLM, $19.7 \pm$ 7.2 cups; $1 \mathrm{D}, 16 \mathrm{LM}, 13.2 \pm 5.2$ cups; $10 \mathrm{D}, 0 \mathrm{LM}, 9.3 \pm$ 4.4 cups; $10 \mathrm{D}, 16 \mathrm{LM}, 11.2 \pm 2.7$ cups).

The animals retrieved a greater percentage of target caches than of control caches under all conditions, as can be seen in Figure 2. A three-way analysis of variance (ANOVA) of the effects of retrieval interval and visual environment on the percentage of targets versus controls retrieved revealed no effect of interval $[F(1,5)=4.21$, $p=.095]$ or visual environment $[F(1,5)=5.88, p=$ $.060]$, only an effect of type of site searched $[F(1,5)=$ $17.19, p=.009]$.

The percentage of targets retrieved was affected by both retention interval $[F(1,5)=12.63, p=.016]$ and visual environment $[F(1,5)=12.28, p=.017]$, with no significant interaction between these effects $[F(1,5)=$
$1.19, p=.224]$. Planned comparisons of percent of targets retrieved at the two retrieval intervals revealed no differences between the $0 \mathrm{LM}$ and $16 \mathrm{LM}$ conditions after a $1 \mathrm{D}$ retention interval $[F(1,5)=0.004, p=.95]$. After the $10 \mathrm{D}$ retention interval, the difference between retrieval in the $0 \mathrm{LM}$ and $16 \mathrm{LM}$ conditions approached significance $[F(1,5)=6.162, p=.056]$. The retrieval of controls was not affected by either retention interval $[F(1,5)=0.52, p=.501]$ or visual environment $[F(1,5)=$ $0.40, p=.555]$, nor did these factors interact $[F(1,5)=$ $0.23, p=.651]$. Comparisons of landmark condition effects at the two retention interval levels also showed no significant effects [ $1 \mathrm{D}$ comparison, $F(1,5)=0.001, p=$ $.979 ; 10 \mathrm{D}$ comparison, $F(1,5)=0.501, p=.511]$.

We then analyzed the initial searches of animals, using hit percentages. Figure 3 shows the proportion of hits directed at each site type by condition. To establish whether initial searches were random or directed, we first compared searches in cups for the combined categories of targets, neighbors, and controls and compared that with searches of uncategorized sites. Of the 63 searches that constituted hits, pooled across all animals and conditions, 61 were in the combined category of targets, neighbors, and controls. Two searches occurred in uncategorized sites: One of these was a cup next to a control site; the other was isolated. On the average, of the 128 cups in the arena, 30 of them were sites consisting of targets, neighbors, and controls, and 98 cups were uncategorized. A chi-square test of the probability of the outcome of $2 / 63$ searches occurring in the cups that consisted of 98/128 of the cups in the arena shows that this result was unlikely to occur by chance $\left(\chi^{2}=67.145, p<.0001\right)$.

An overall two-way ANOVA of the percent of target hits in initial searches did not reveal any significant effects [effect of retention interval, $F(1,5)=0.430, p=.541$; effect of landmarks, $F(1,5)=3.567, p=.118$; interaction, $F(1,5)=4.217, p=.095$ ]; see Figure 4. Planned comparisons at the two retention intervals showed that there was no difference in hits of targets at the $1 \mathrm{D}$ retention interval $[F(1,5)=0.013, p=.913]$. However, at the $10 \mathrm{D}$ retention interval, a significant effect of landmarks was found $[F(1,5)=10.554, p=.023]$. No statistically significant effects were found in hit percentages of controls [retention interval, $F(1,5)=0.401, p=.550$; landmarks, $F(1,5)=0.445, p=.530$; interaction, $F(1,5)=$ $3.776, p=.110]$. Comparisons at the two retention intervals also revealed no significant effects [1D, $F(1,5)=$ $.302, p=.606 ; 10 \mathrm{D}, F(1,5)=2.893, p=.150]$.

\section{DISCUSSION}

The overall retrieval of more target caches than control caches indicates that the kangaroo rats remembered the location of their caches and used this memory to relocate them. It is unlikely that controls were located by using memory, because an average of 3 months had elapsed between the use of a site as a cache and its reuse as a control. In addition, the data strongly suggest that the kangaroo 


\section{A) Targets}

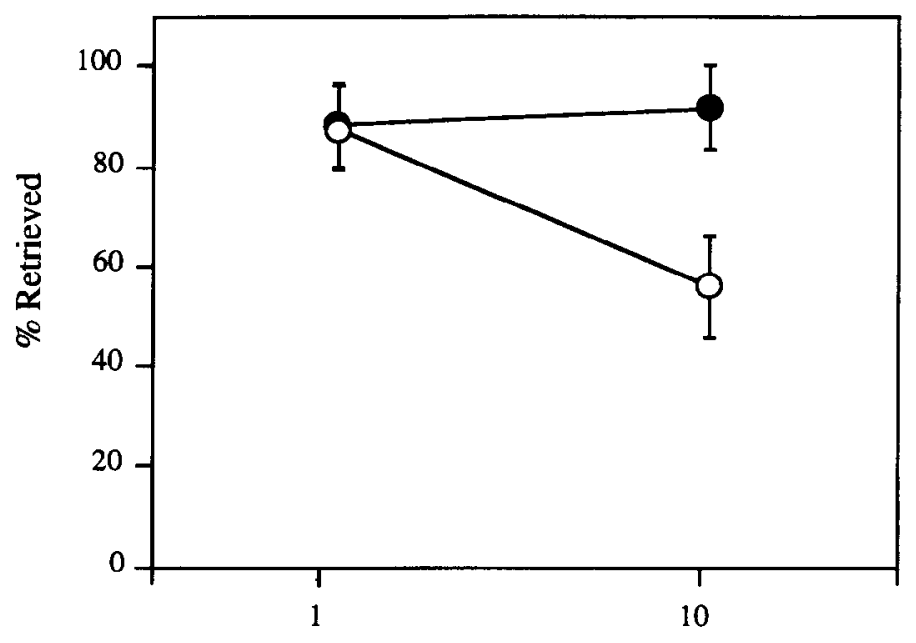

Cache Retrieval Interval (days)

\section{B) Controls}

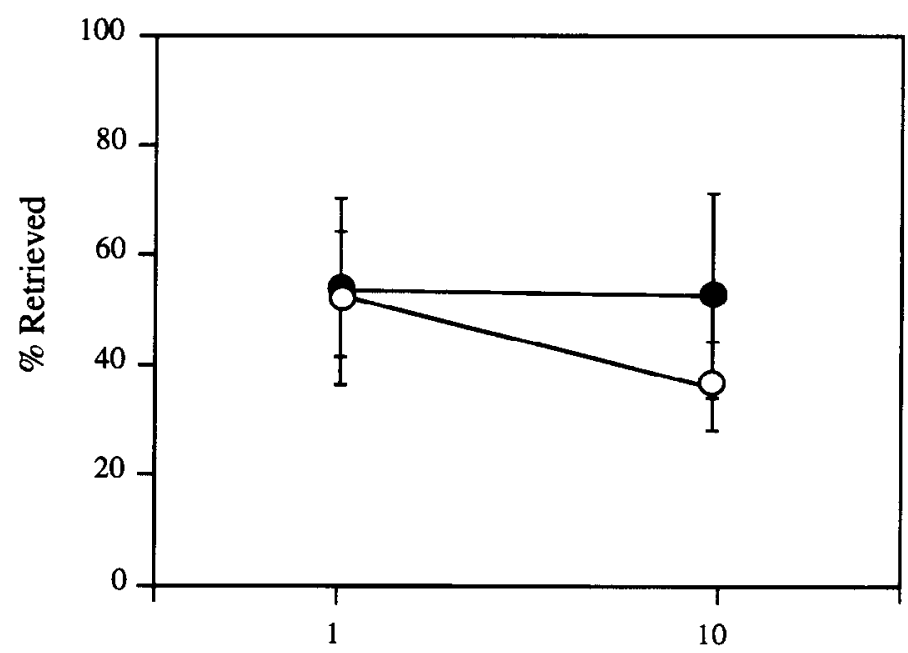

Cache Retrieval Interval (days)

Figure 2. Percent retrieval of caches recovered during the cache retrieval session $(N=6)$. Open circles represent the average $( \pm S E)$ performance in the 0-landmark condition and the filled circles represent the average $( \pm S E)$ performance in the 16-landmark condition. (A) Percentage of targets retrieved. (B) Percentage of controls retrieved.

rats treated targets and controls differently. Note that all cups adjacent to control sites were classified as uncategorized sites in the hit analyses. These sites were almost never searched by kangaroo rats: The percentage of initial searches of uncategorized sites per condition was only 0.8 . By comparison, the percentage of initial searches of neighbors (sites adjacent to target caches) per condition was 18.4. Finally, initial searches of neighbors occurred in all conditions, but searches of uncategorized sites occurred only in the most demanding of the four conditions, the 10D, 0LM condition (Figure 3).

Fewer caches were made in the 0LM conditions than in the $16 \mathrm{LM}$ conditions. This might have affected retrieval accuracy; accuracy in the 1D, OLM condition was not affected by this difference, however. In addition, equal numbers of seeds were stored across conditions and 


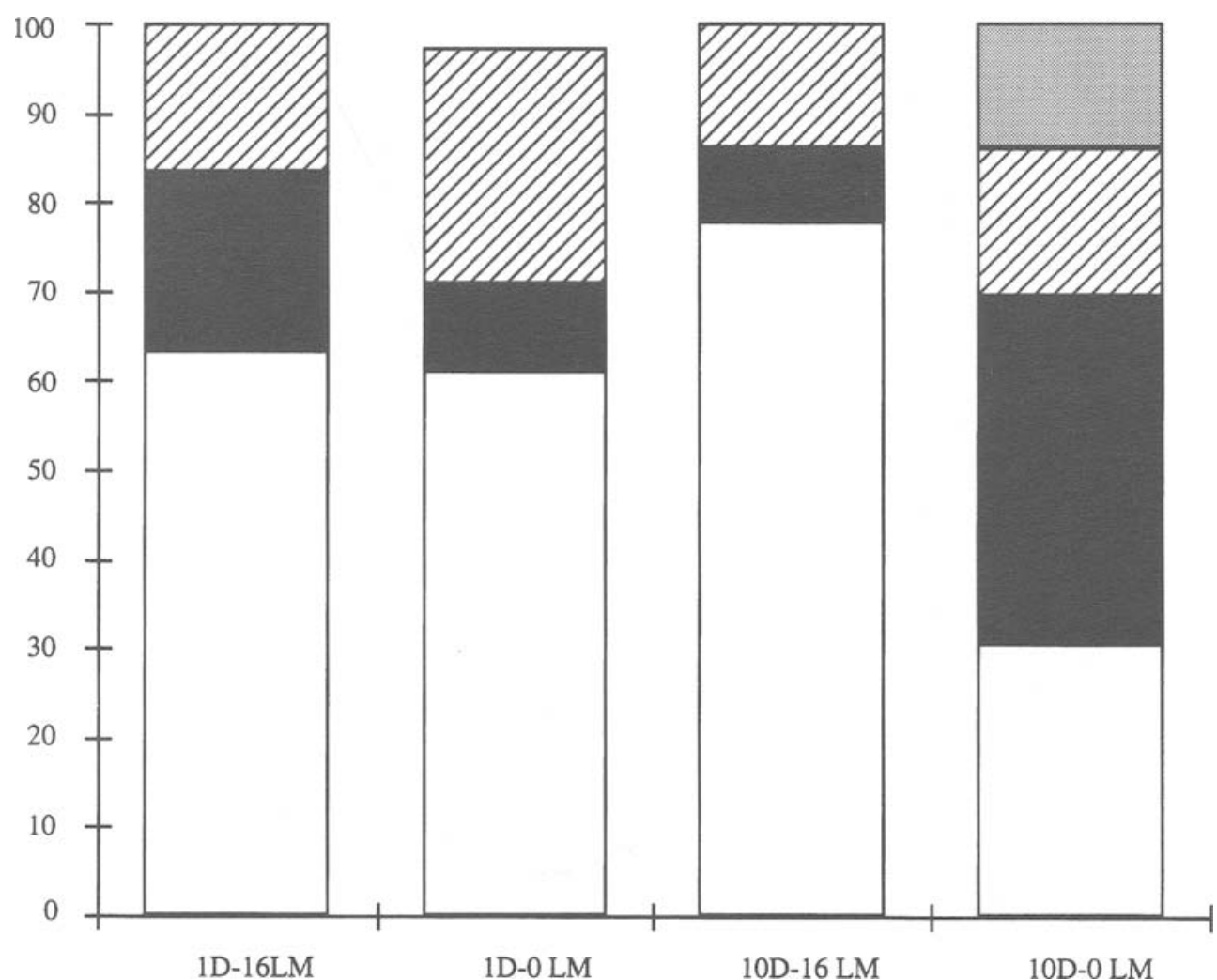

Figure 3. Distribution of hits during a retrieval session directed at site types. Hit percentage is defined as the proportion of sites of a certain type searched in the beginning of a retrieval session, up to the total number of caches available at the beginning of that session. Open bars are proportion of searches in target caches, filled bars are proportion of searches in controls, hatched bars are searches of neighboring sites to targets, and gray bar is searches in all other uncategorized cups in the arena.

the hunger level of animals across conditions should have been the same; thus, motivation to search for caches should also have been equal across conditions.

The accuracy of cache recall was manipulated via the two parameters that we predicted would interact to affect cache retrieval accuracy: an increased delay between caching and retrieval, and the presence of a unique visual array of landmarks. We used two different methods to analyze cache retrieval accuracy: hit percentage of targets and percent recovery of targets. The percentage of targets hit was not different in the two landmark conditions in the $1 \mathrm{D}$ retention interval. In the $10 \mathrm{D}$ interval, however, the percentage of targets hit was higher in the $16 \mathrm{LM}$ condition than in the $0 \mathrm{LM}$ condition. The results of the analyses using percent recovery of targets was similar to the hit analyses, with the difference between the $16 \mathrm{LM}$ and $0 \mathrm{LM}$ conditions in the $10 \mathrm{D}$ conditions approaching significance.

There were no statistically significant effects of the manipulations on recovery of control caches. However, the percentage of controls recovered in the $10 \mathrm{D}, 0 \mathrm{LM}$ condition was lower than in the other conditions. Perhaps failing to find or remember their own caches led to a decreased drive to search for caches. This is supported by the decrease in overall number of sites searched in this condition. The drop in activity level and control searches in this condition does not invalidate the overall results, however. In the hit analysis, while percentage of targets hit was lower in the $10 \mathrm{D}, 0 \mathrm{LM}$ than in the $10 \mathrm{D}, 16 \mathrm{LM}$ condition, the percentage of control hits was higher, and this shows the opposite effect (Figure 4).

The animals used for the test phase of the experiment were those most likely to cache reliably. Our elimination procedure produced a mix of one-third male and two-thirds female subjects in this experiment. A previous study of cache retrieval in Merriam's kangaroo rats showed no sex differences in retrieval accuracy (Jacobs, 1992). However, in a study of sex differences in the desert kangaroo rat (Dipodomys deserti), Langley found that female kangaroo rats were able to use intra-apparatus landmark cues to locate a goal, whereas male kangaroo rats did not locate the goal by using these cues (Langley, 1994). In addition, Williams, Barnett, and Meck (1990) found that female Norway rats relied more heavily on visual landmarks to orient themselves to food locations than did males (although in that study the landmarks were extra-apparatus). Thus, if female Merriam's kangaroo rats particularly attend to the kind of intra-apparatus landmarks that we 


\section{A) Targets}

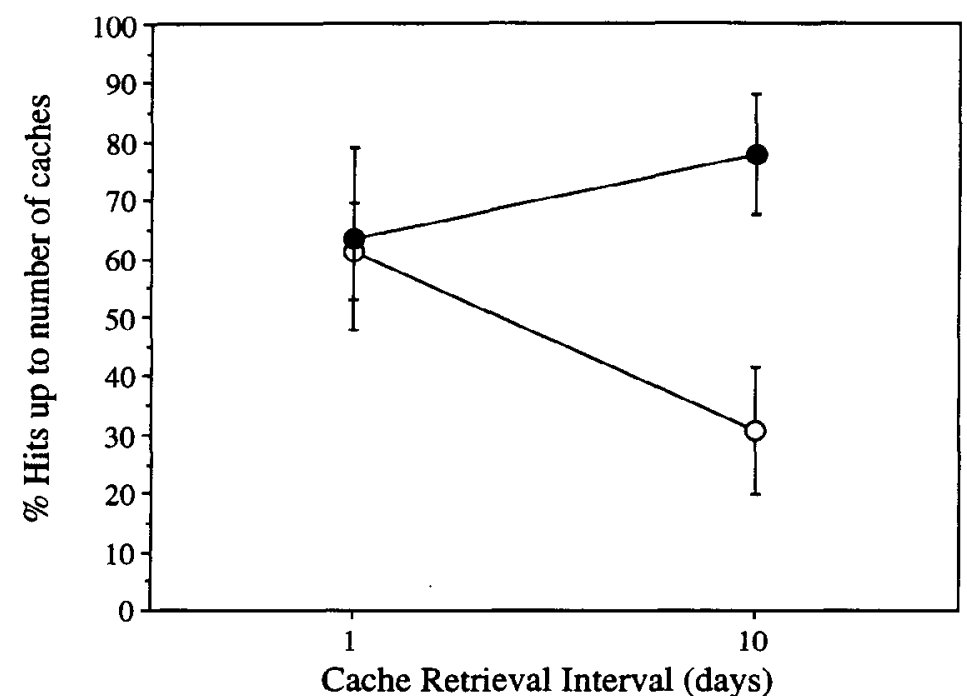

\section{B) Controls}

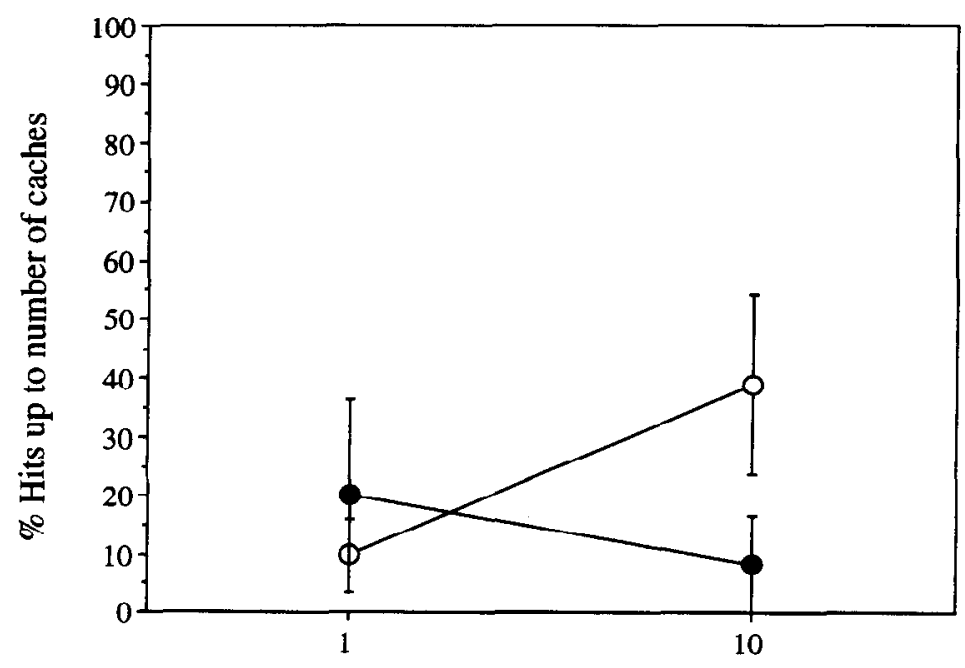

Cache Retrieval Interval (days)

Figure 4. Searches during retrieval sessions directed toward target and control sites. Hit percentage is defined as the proportion of sites of a certain type dug in the beginning of a retrieval session, up to the total number of caches available at the beginning of that session. Open circles represent the average $( \pm S E)$ performance in the 0-landmark condition, and the filled circles represent the average $( \pm S E)$ performance in the 16-landmark condition. (A) Hit percentage of targets. (B) Hit percentage of controls.

chose to manipulate in our experiment, the size of our effect could have been influenced by the high proportion of female subjects. Future studies done with artificial tasks may be able to test the effects of landmark manipulations without the necessity of subject elimination, and with equal proportions of males and females.

We chose to manipulate intra-apparatus cues in our experiment. In other studies, intra-apparatus cues have been more salient than extra-apparatus cues in a discrimination task (e.g., Chamizo, Sterio, \& Mackintosh, 1985; March, Chamizo, \& Mackintosh, 1992). Langley's foodfinding study, with desert kangaroo rats, yielded the opposite effect: animals did not rely primarily on intra-apparatus cues, but instead oriented themselves toward goals by using extra-apparatus cues (Langley, 1994). Such differences in cue saliency may derive from the type of 
cue rather than its position relative to the maze, however. In the present experiment, we chose our landmarks from a large pool of uniquely shaped objects, such as rocks and flowers. In contrast, the intra-apparatus objects in Langley's experiment were symmetrically placed and identically shaped objects (four wood blocks). Perhaps either the variety of objects, and/or their trial-unique spatial configurations, increased the saliency of our intraapparatus cues and hence their utility for predicting the location of caches. In addition to the type of landmarks, our use of trial-unique landmarks in habituation and pretest trials may have affected how the kangaroo rats responded to the testing conditions. For example, they may have become more attentive to the landmarks once they learned that the landmarks were trial unique and hence useful in predicting the location of caches for retrieval. Thus spatial memory for caches could be influenced both by the kind of landmarks available and by the training procedures.

How 16 landmarks helped maintain memory for cache sites over many days remains to be determined. On the one hand, kangaroo rats could use landmarks to enhance their recall of caches through an increased precision of encoding cache location. If, for example, they use landmarks to encode locations by calculating vectors between cache and landmark sites (Collett, Cartwright, \& Smith, 1986), accuracy would decrease in the 10D, 0LM condition because the arena is bare and precise coding is thus difficult. This would not be surprising, given that many scatter hoarders use nearby landmarks to calculate locations of caches or goal sites (Balda \& Turek, 1984; Kamil \& Jones, 1997; Vander Wall, 1982, 1991). Similarly, laboratory rats swimming in the Morris water maze concentrate their search more precisely around the hidden platform when there are more spatial cues in the environment (see the comparison of data from Pellymounter, Smith, \& Gallagher, 1987, and Rapp, Rosenberg, \& Gallagher, 1987, in Leonard \& McNaughton, 1990). This hypothesis of precision, however, does not explain why the value of landmarks for retrieval accuracy would increase with time since caching.

An alternative hypothesis is that trial-unique landmarks enhance memory after a long interval by reducing proactive interference from previous trials. Reducing interference by changing contextual cues, such as maze color or floor texture between trials, reduces or eliminates forgetting in laboratory rats, even $\mathbf{4 0}$ days after training (Zentall, 1970). Although this study only showed a reduction in retroactive interference, changing the spatial array of objects should also reduce proactive interference. This has been found in radial arm maze experiments in which proactive interference is reduced when intra- or extra-maze cues were changed between trials (Cohen et al., 1994). In addition, food-storing birds tested in food-finding tasks cannot remember the baited location unless they are provided with trial-unique cues (Brodbeck et al., 1992). In our experiment, as well as Brodbeck's, these trial-unique spatial cues may have been acting to reduce proactive interference between repeated recall tests in the same environment. Without unique spatial cues, interference may blur the distinction between events in the same or similar environments, making it difficult to distinguish between these events over long time periods.

Retrieval intervals have also been found to be important in proactive interference in Norway rats, with longer retrieval intervals producing more interference (Roberts \& Dale, 1981). Although we did not find retrieval interval by itself to cause deterioration in recall, we did find that the retrieval interval acted with the absence of landmarks to reduce cache recovery accuracy. Our failure to find a reduction in accuracy with the retrieval interval manipulation alone may have stemmed from our choice of 10 days as our longest interval. Previous tests of cache memory in the black-capped chickadee showed no deterioration of memory before 28 days (Hitchcock \& Sherry, 1990). This is the shortest retrieval interval after which a reduction in cache recovery accuracy has been observed in the laboratory. And although we cannot directly compare our results from a mammalian species caching in two dimensions to those from an avian species caching in three dimensions, it is nonetheless striking that we could produce a reduction in accuracy in as short an interval as 10 days by forcing animals to cache in a bare environment. This suggests that a profitable approach for future research may be to manipulate the visual environment of the caching session, rather than the length of the retrieval interval, to measure the memory capacity of scatter hoarders.

Landmarks clearly play a role in memory for cache locations since scatter-hoarding animals appear to rely on the relationship between their caches and nearby landmarks to return to specific locations (Balda \& Turek, 1984; Bennett, 1993; Vander Wall, 1982, 1991). Yet what has not been understood is whether landmarks are necessary for remembering cache locations. Although our results show that landmarks are not necessary over short retention intervals, they appear to play a crucial role in sustaining the memory over longer intervals. In our experiment, we manipulated the presence of landmarks and the trial-unique arrangement together, however; future research should explore how these factors can be separated so that their independent roles in influencing memory for caches locations can be evaluated.

Finally, our finding that trial-unique landmarks are important for sustaining spatial memory over time is similar to findings from tests of spatial memory in laboratory rats (Cohen et al., 1994; Zentall, 1970). However, scatterhoarding species can remember locations in space longer than other species (Hilton \& Krebs, 1990; Krebs, Healy, $\&$ Shettleworth, 1990), and can rely on a different hierarchy of cues to relocate spatial locations from that used by nonstoring species (Brodbeck, 1994; Clayton \& Krebs, 1993). Thus it is not yet clear whether there is a qualitative difference in the effect of landmarks and test latency 
on recall accuracy in scatter-hoarding species of rodents and larder-hoarding species such as the laboratory rat.

In conclusion, this study shows that intra-apparatus landmarks play a significant role in the memory for caches of kangaroo rats willing to cache frequently when there is a lengthy delay between caching and recovery. These landmarks may be important in memory either because they allow the animal to calculate the precise location of each cache, or because they provide a unique spatial array that the animal can use to discriminate between caches made over repeated trials in the same arena, reducing interference from previous trials. Future experiments arising from the present study will help determine what information scatter-hoarding animals are storing in memory about the visual scenes around their caches and further our understanding of how temporal and spatial information are integrated into memory.

\section{REFERENCES}

Andersson, M., \& Krebs, J. (1978). On the evolution of hoarding behaviour. Animal Behaviour, 26, 707-711.

BALDA, R. P, \& KAMIL, A. C. (1989). A comparative study of cache recovery by three corvid species. Animal Behaviour, 38, 486-495.

BALDA, R. P., \& KAMIL, A. C. (1992). Long-term spatial memory in Clark's nutcracker Nucifraga columbiana. Animal Behaviour, 44, 761-769.

BaldA, R. P., \& TUREK, R. J. (1984). The cache-recovery system as an example of memory capabilities in Clark's nutcracker. In H. L. Roitblat, T. G. Bever, \& H. S. Terrace (Eds.), Animal cognition (pp. 513532). Hillsdale, NJ: Erlbaum.

BENNETr, A. T. D. (1993). Spatial memory in a food storing corvid: I. Near tall landmarks are primarily used. Joumal of Comparative Physiology A, 173, 193-207.

BRoDBECK, D. R. (1994). Memory for spatial and local cues: A comparison of a storing and a nonstoring species. Animal Learning \& Behavior, 22, 119-133.

Brodbeck, D. R., BUrack, O. R., \& SHETTLEWorTh, S. J. (1992). Onetrial associative memory in black-capped chickadees. Journal of Experimental Psychology: Animal Behavior Processes, 18, 12-21.

Chamizo, V. D., Sterio, D., \& Mackintosh, N. J. (1985). Blocking and overshadowing between intra-maze and extra-maze cues: A test of the independence of locale and guidance learning. Quarterly Journal of Experimental Psychology, 37B, 235-253.

Clayton, N. S. (1995). Comparative studies of food-storing, memory, and the hippocampal formation in parids. Hippocampus, 5, 499-510.

Clayton, N. S., \& KREBS, J. R. (1993). Memory for spatial and local cues in food-storing and non-storing birds. Journal of Comparative Physiology A, 174, 371-379.

COHEN, J. S., ReID, S., \& CHEW, K. (1994). Effects of varying trial distribution, intra- and extramaze cues, and amount of reward on proactive interference in the radial maze. Animal Learning \& Behavior, 22, 134-142.

Collett, T. S., Cartwright, B. A., \& Smith, B. A. (1986). Landmark learning and visuo-spatial memories in gerbils. Journal of Comparative Physiology, 158, 835-851.

DALY, M., JACOBS, L. F., \& WILSON, M. I. (1992). Scatter-hoarding by kangaroo rats (Dipodomys merriami) and pilferage from their caches. Behavioral Ecology, 3, 102-111.

HAMPton, R. R., \& SHERry, D. F. (1994). The effects of cache loss on choice of cache sites in black-capped chickadees. Behavioral Ecology, 5, 44-50.

HizTon, S. C., \& Krebs, J. K. (1990). Spatial memory of four species of Parus: Performance in an open-field analogue of a radial maze. Quarterly Journal of Experimental Psychology, 42B, 345-368.
HITCHCOCK, C. L., \& SHERRY, D. F. (1990). Long-term memory for cache sites in the black-capped chickadee. Animal Behaviour, 40, 701-712.

JACOBS, L. F. (1992). Memory for cache locations in Merriam's kangaroo rats. Animal Behaviour, 43, 585-593.

JACOBS, L. F. (1995). The ecology of spatial cognition: Adaptive patterns of hippocampal size and space use in wild rodents. In E. Alleva, A. Fasolo, H.-P. Lipp, \& L. Nadel (Eds.), Studies of the brain in naturalistic settings (NATO Advanced Studies Institute Series D, Vol. 82, pp. 301-322). Dordrecht: Kluwer.

JACOBS, L. F., \& LiMAN, E. R. (1991). Grey squirrels remember the locations of buried nuts. Animal Behaviour, 41, 103-110.

Jenkins, S. H., \& PETERS, R. A. (1992). Spatial patterns of food storage by Merriam's kangaroo rats. Behavioral Ecology, 3, 60-65.

KAMIL, A. C., \& JoNES, J. E. (1997). The seed-storing corvid Clark's nutcracker learns geometric relationships among landmarks. Nature, 390, 276-279.

Krebs, J. R., Healy, S. D., \& Shettleworth, S. J. (1990). Spatial memory of Paridae: Comparison of a storing and a non-storing species, the coal tit, Parus ater, and the great tit, P. major. Animal Behaviour, 39, $1127-1137$.

LANGLEY, C. M. (1994). Spatial memory in the desert kangaroo rat (Dipodomys deserti). Journal of Comparative Psychology, 108, 3-14.

LEONARD, B., \& MCNAughton, B. L. (1990). Spatial representation in the rat: Conceptual, behavioral, and neurophysiological perspectives. Hillsdale, NJ: Erlbaum.

March, J., Chamizo, V. D., \& Mackintosh, N. J. (1992). Reciprocal overshadowing between intra-maze and extra-maze cues. Quarterly Journal of Experimental Psychology, 45B, 49-63.

MorRIs, D. (1962). The behaviour of the green acouchi (Myoprocta pratti) with special reference to scatter hoarding. Proceedings of the Zoological Society of London, 139, 701-732.

Pellymounter, M. A., Smith, M. Y., \& Gallagher, M. (1987). Spatial learning impairments in aged rats trained with a salient configuration of stimuli. Psychobiology, 15, 248-254.

RapP, P. R., Rosenberg, R. A., \& Gallagher, M. (1987). An evaluation of spatial information processing in aged rats. Behavioral Neuroscience, 101, 3-12.

ReynolDs, H. G. (1958). The ecology of the Merriam kangaroo rat (Dipodomys merriami Mearns) on the grazing land of southern Arizona. Ecology Monographs, 28, 111-117.

RoberTs, W. A., \& DALE, R. H. I. (1981). Remembrance of places lasts: Proactive inhibition and patterns of choice in rat spatial memory. Learning \& Motivation, 12, 261-281.

SHETTLEWORTH, S. J. (1995). Memory in food storing birds: From the field to the Skinner box. In E. Alleva, A. Fasolo, H.-P. Lipp, \& L. Nadel (Eds.), Studies of the brain in naturalistic settings (NATO Advanced Studies Institute Series D, Vol. 82, pp. 159-192). Dordrecht: Kluwer.

Thompson, D. C., \& Thompson, P. S. (1980). Food habits and caching behavior of urban grey squirrels. Canadian Journal of Zoology, 58, $701-710$.

VANDER WALL, S. B. (1982). An experimental analysis of cache recovery in Clark's nutcracker. Animal Behaviour, 30, 84-94.

VANDER WALL, S. B. (1990). Food hoarding in animals. Chicago: University of Chicago Press.

VANDER WALL, S. B. (1991). Mechanisms of cache recovery by yellow pine chipmunks. Animal Behaviour, 41, 851-864.

VANDER WALl, S. B., \& Hutchins, H. E. (1983). Dependence of Clark's nutcrackers (Nucifraga columbiana) on conifer seeds during the postfledgling period. Canadian Field Naturalist, 97, 208-214.

Williams, C. L., Barnett, A. M., \& Meck, W. H. (1990). Organizational effects of early gonadal secretions on sexual differentiation in spatial memory. Behavioral Neuroscience, 104, 84-97.

ZENTALL, T. R. (1970). Effects of context change on forgetting in rats. Journal of Experimental Psychology, 86, 440-448.

(Manuscript received August 28, 1997; revision accepted for publication March 9, 1998.) 